\title{
Notizen und Kurzbeiträge
}

http://doi.org/10.1515/bd-2019-0031

\section{Erfolgreich evaluiert: Die Technische Informationsbibliothek (TIB) leistet herausragende Arbeit}

\author{
Leibniz-Senat empfiehlt die Fortführung der Förderung der \\ Bibliothek in der Leibniz-Gemeinschaft
}

Der Senat der Leibniz-Gemeinschaft hat am 27. November 2018 die weitere finanzielle Förderung der TIB - Leibniz-Informationszentrum Technik und Naturwissenschaften empfohlen. Grundlage für die Entscheidung ist das Ergebnis einer im Februar 2018 durchgeführten externen Evaluierung, der sich jede LeibnizEinrichtung alle sieben Jahre stellen muss. Vor Ort in Hannover überprüften die Expertinnen und Experten die Bibliothek zwei Tage lang auf Herz und Nieren. Das Ergebnis: Die TIB leistet hervorragende Arbeit. „Über die positive Evaluierung freuen wir uns sehr. Sie bestätigt die Strategie der TIB, sich als modernes Informationszentrum für die Digitalisierung von Wissenschaft und Technik zu positionieren und dies durch exzellente Forschung mit potenzialträchtigen Anwendungen umzusetzen“, erklärt TIB-Direktor Prof. Dr. Sören Auer.

\section{Von der klassischen Bibliothek zum digitalen Informationszentrum}

Der Leibniz-Senat betonte in seiner Stellungnahme, dass die Bibliothek den durch die Digitalisierung bedingten tiefgreifenden Transformationsprozess von einer klassischen Bibliothek hin zu einem zunehmend digitalen Informationszentrum in überzeugender Weise gestalte. Der Senat weist in diesem Zusammenhang darauf hin, dass die TIB in den letzten Jahren ihren Bestand systematisch um audiovisuelle Medien, Forschungsdaten und wissenschaftliche Software erweitert habe. Außerdem werde der exzellente bibliothekarische Kernbereich der TIB inzwischen durch vielfältige, überwiegend sehr gute Forschungs- und Entwicklungsarbeiten ergänzt. Diese Arbeiten seien wichtig, damit die TIB innovative Angebote und Produkte für die Wissenschaft bereitstellen könne. Positiv 
hervorgehoben wird außerdem, dass der neue Direktor der TIB, ein ausgewiesener Informatiker, erstmals gemeinsam mit der Universität Hannover auf eine Professur berufen worden sei. Seine Aufgabe sei es nun, die Forschungsarbeiten und die Entwicklung der digitalen Dienste der TIB noch enger aufeinander zu beziehen. Dafür sieht der Senat sehr gute Grundlagen.

Der Senat empfiehlt Bund und Ländern, die gemeinsame Förderung der TIB fortzuführen. Anschließend überprüft die Gemeinsame Wissenschaftskonferenz (GWK), ob die Voraussetzungen für eine gemeinsame Förderung der Einrichtung durch Bund und Länder nach wie vor bestehen.

\title{
Weitere Informationen im Internet:
}

- Zur Stellungnahme des Senats der Leibniz-Gemeinschaft zur TIB: https:// www.leibniz-gemeinschaft.de/ueber-uns/evaluierung/das-evaluierungsverfahren-des-senats/senatsstellungnahmen

- Zur Pressemitteilung der Leibniz-Gemeinschaft: https://www.leibniz-gemeinschaft.de/medien/presse/pressemitteilungen/details/article/leibniz_einrichtungen_in_berlin_hannover_frankfurt_am_main_trier_und_dresden_ evaluiert_100003743

\section{Hohe Auszeichnung: Prof. Dr. Sören Auer erhält renommierten ERC Consolidator Grant}

\author{
Direktor der TIB erhält zwei Millionen Euro für sein \\ Forschungsprojekt zu wissensbasierten Informationsflüssen
}

Prof. Dr. Sören Auer, Direktor der TIB - Leibniz-Informationszentrum Technik und Naturwissenschaften und Professor für Data Science \& Digital Libraries an der Leibniz Universität Hannover, erhält einen der renommierten Consolidator Grants des Europäischen Forschungsrates (ERC).

Für seine Forschungsarbeit mit dem Thema „ScienceGraph - Knowledge Graph based Representation, Augmentation and Exploration of Scholarly Communication“ stellt die Europäische Union Auer in den kommenden fünf Jahren zwei Millionen Euro zur Verfügung. Das Projekt wird im Joint Lab „Data Science \& Open Knowledge“ von der TIB und dem Forschungszentrum L3S der Leibniz Universität angesiedelt sein. 


\section{Revolutionierung der wissenschaftlichen Zusammenarbeit}

Wie kann der Umgang mit Informationen, Daten und Wissen verbessert und effektiver gestaltet werden? Wie können Wissen und Informationen angesichts des enormen technologischen Fortschritts digital vernetzt werden, um sie künftig besser maschinell nutzbar zu machen? Wie kann der Wandel von dokumentenzentriertem Wissensaustausch in Wissenschaft und Forschung hin zu stärker wissensbasierten Informationsflüssen erfolgen? Mit diesen Fragen beschäftigen sich Auer und sein Team an der TIB und dem Forschungszentrum L3S in Hannover.

„Wissensaustausch in der Forschung erfolgt heutzutage noch immer über Dokumente. Genau genommen hat sich die Art und Weise, wie wissenschaftliche Erkenntnisse weitergegeben werden, seit Jahrhunderten nicht grundlegend verändert. Und das, obwohl wir ein immer schnelleres Tempo bei der Entwicklung von neuen digitalen Technologien erleben“, so Auer. „Mit der Anerkennung des ERC haben wir nun die Möglichkeit, unsere Forschungen zu einem stärker bedeutungsorientierten Wissensaustausch durch semantische Datenbanken, sogenannte Wissensgraphen, $\mathrm{zu}$ intensivieren, und damit Forschung effektiver zu gestalten und in das Zeitalter der Digitalisierung zu führen“, freut sich Auer.

Die von der Europäischen Union mit einer Gesamtsumme von 573 Millionen Euro finanzierten Wissenschaftspreise sollen Spitzenwissenschaftler bei ihren visionären Grundlagenforschungen unterstützen. 2018 wurden europaweit 291 der prestigeträchtigen ERC Consolidator Grants vergeben. Bei 2.389 Bewerbungen entspricht dies einer Erfolgsquote von lediglich zwölf Prozent.

Weitere Informationen im Internet Vorarbeiten zum Forschungsprojekt:

- http://tib.eu/thq7

- http://projects.tib.eu/orkg 


\section{Neue Zeitschrift „Quantitative Science Studies" gegründet}

\section{TIB unterstützt Journal Flipping}

Die Fachgesellschaft International Society for Scientometrics and Informetrics (ISSI) gibt mit Partnern die Neugründung der Open-Access-Zeitschrift Quantitative Science Studies (QSS) bekannt. Die Technische Informationsbibliothek (TIB) unterstützt diesen Prozess durch eine signifikante Beteiligung an den Transformationskosten.

Eine wesentliche Voraussetzung für die Open-Access-Transformation ist die Bereitstellung bibliothekarischer Erwerbungsmittel für die Finanzierung von Open-Access-Publikationen. Diese Umwandlung von Zeitschriften aus dem bestehenden Subskriptionssystem in Open Access wird als Journal Flipping bezeichnet. „Mit Journal Flipping erfüllt die TIB ihren Auftrag, bestmöglichen Zugang zu wissenschaftlicher Literatur zu schaffen. Wir erweitern unsere Tätigkeiten, setzen unsere Mittel strategisch ein und bauen auf umfangreichen Erfahrungen bei Konsortialbildungen und Open-Access-Diensten auf“, so Irina Sens, stellvertretende Direktorin. Die TIB hat sich bereiterklärt, für die ersten drei Jahre artikelbezogene Kosten der Zeitschrift QSS zu übernehmen.

Die gemeinsame Pressemitteilung (in englischer Sprache) der beteiligten Partner finden Sie hier: http://issi-society.org/blog/posts/2019/january/theinternational-society-for-scientometrics-and-informetrics-ends-support-forjournal-of-informetrics-launches-new-open-access-journal-quantitative-sciencestudies.

Nähere Informationen zur Rolle der TIB haben wir für Sie im Blog zusammengestellt: https://blogs.tib.eu/wp/tib/2019/01/14/unterstuetzung-journal-flippingqss.

Für weitere Fragen zu Open Access und Jounal Flipping steht Ihnen Marco Tullney (marco.tullney@tib.eu), Leiter Publikationsdienste und Koordinator Open Access, zur Verfügung.

\section{Kontakt:}

Dr. Sandra Niemeyer, Technische Informationsbibliothek (TIB)

Pressereferentin / Kommunikation und Marketing

Welfengarten 1 B, 30167 Hannover

Tel.: (0511) 762 2772, E-Mail: sandra.niemeyer@tib.eu 


\section{Fördermittel für Wissenschaftsanalyse- Projekt Q-Aktiv}

\section{BMBF unterstützt das Kooperationsprojekt in der Förderlinie "Quantitative Wissenschaftsforschung”}

Im Projekt Q-Aktiv steht die Erforschung von Wissenschaftskonvergenzen und -dynamiken im Fokus. Die Projektpartner Christian-Albrechts-Universität zu Kiel (CAU), ZB MED - Informationszentrum Lebenswissenschaften und ZBW Leibniz-Informationszentrum Wirtschaft erhalten dafür Drittmittel in Höhe von rund 650.000 Euro. Die Laufzeit beträgt drei Jahre. Das Bundesministerium für Bildung und Forschung (BMBF) finanziert das Projekt in der Förderlinie „Quantitative Wissenschaftsforschung“ im Schwerpunkt „Wissenschafts- und Hochschulforschung“. Der offizielle Kick-off aller 24 Projekte der Förderlinie fand am 23. November 2018 im Fraunhofer-Forum in Berlin statt.

Der Projektleiter von Q-Aktiv Prof. Carsten Schultz, Professor für Technologiemanagement und Innovationsforschung an der CAU, erläutert die Hintergründe des Projekts: „Wissenschaftliche Forschungsprozesse haben sich auf Grund moderner Forschungsverfahren und in Folge der Digitalisierung in den letzten Jahren stark verändert. Das Publikationsaufkommen ist gewaltig angewachsen. Forschungsbereiche arbeiten zunehmend problemorientiert und transdisziplinär. Eine Analyse der Prozesse wird bisher jedoch kaum methodisch quantitativ unterstützt. Mit unserem Ansatz versuchen wir bei der strategischen Frühaufklärung zu helfen.“

Hier setzt das Projekt Q-Aktiv an. Die Kurzform steht für „Quantitative Analyse der Dynamik des wissenschaftlichen, wirtschaftlichen und gesellschaftlichen Impacts von Forschungsaktivitäten und -netzwerken“. Die Projektpartner analysieren wissenschaftliche Innovationsprozesse von der Idee bis hin zur Etablierung in der Wissenschaft oder der praktischen Anwendung etwa als Patent. Sie stützen sich dabei auf insgesamt 10 Millionen Artikel und Patentschriften aus den Lebensund Wirtschaftswissenschaften. Ziel ist es, die Determinanten der Wissens- und Technologiekonvergenzen zu ermitteln sowie die Faktoren, die zum Gelingen oder Scheitern wissenschaftlicher Ideen führen, besser zu verstehen. Die Forschenden im Projekt wenden dabei unter anderem Text- und Data-Mining-Verfahren, Netzwerkanalysen sowie Methoden der Informatik und Sozialwissenschaften an.

Prof. Konrad Förstner, Leiter von Q-Aktiv bei ZB MED, ist sicher: „Im Ergebnis wird Q-Aktiv einen zuverlässigen und umfassenden Werkzeugkasten zur Analyse und Prognose der Dynamik des Wissenschafts- und Innovationssystems liefern.“ 
Das Instrumentarium wird Unternehmen sowie Forschungseinrichtungen und -politik in Entscheidungsprozessen unterstützen, zum Beispiel bei der Anmeldung von Patenten basierend auf wissenschaftlichen Publikationen.

\section{Hintergrund-Informationen}

\section{Christian-Albrechts-Universität zu Kiel}

Als einzige Volluniversität in Schleswig-Holstein, mit mehr als 27.000 Studierenden und über 2.000 Wissenschaftlerinnen und Wissenschaftlern, bietet die Christian-Albrechts-Universität zu Kiel (CAU) ein fachübergreifendes akademisches Umfeld. Zwischen den Disziplinen ihrer acht Fakultäten entstehen permanent dynamische Schnittstellen, die sich in der Lehre und in einem integrativen Forschungsprofil widerspiegeln. Im Jahr 2008 entstanden auf der Grundlage intensiver Zusammenarbeit vier Forschungsschwerpunkte: Lebenswissenschaften, Meereswissenschaften, Nanowissenschaften und Oberflächenforschung sowie Gesellschaft, Umwelt, Kultur im Wandel. Jeder Schwerpunkt bündelt die Expertisen verschiedener Fakultäten aus den Natur-, Sozial-, Technik-, Rechts- und Geisteswissenschaften. An der CAU entwickelte Ideen und Erfindungen tragen zum technologischen und gesellschaftlichen Fortschritt bei. Die Forschung des Instituts für Betriebswirtschaftslehre befasst sich überwiegend mit dem Management von Innovationen. Forschungsschwerpunkt des Instituts für Betriebswirtschaftslehre ist die empirische Analyse des Wandels von Organisationen. Dies umfasst das Management von technologiebasierten Innovationsprozessen, die zunehmende Vernetzung der Wertschöpfung und die Reaktion des Marketing auf veränderte gesellschaftliche Bedürfnisse.

Mehr Informationen unter www.uni-kiel.de/de.

\section{ZB MED - Informationszentrum Lebenswissenschaften}

ZB MED - Informationszentrum Lebenswissenschaften versteht sich als zentrale lebenswissenschaftliche Informationsinfrastruktur für Deutschland und Europa und als treibende Kraft bei der Schaffung einer vernetzten digitalen Wissensbasis. Aufbauend auf seinen einzigartigen Beständen bietet ZB MED forschungsbasierte Möglichkeiten zur Gewinnung von Informationen und Nutzung von Forschungsdaten in den Lebenswissenschaften. Als Dienstleister für die lebenswissenschaftliche Forschung ist ZB MED Partner für alle, die innovative Wege zur verbesserten 
Gewinnung, Verarbeitung und Nutzung von wissenschaftlichen Informationen und Forschungsdaten beschreiten wollen. Dazu bietet das Informationszentrum Literatur, Fachinformationen sowie darauf aufbauende Mehrwertdienste vor allem in digitaler Form über das semantikbasierte Suchportal LIVIVO, über das Publikationsportal PUBLISSO sowie vor Ort in Köln und Bonn an. Im Interesse einer qualitativ hochwertigen Versorgung mit wissenschaftlichen Informationen gehört die Förderung von Open Access zu den zentralen Prioritäten von ZB MED. Zudem betreibt das Informationszentrum anwendungsorientierte Forschung im Bereich „Knowledge Discovery“.

Mehr Informationen unter https://www.zbmed.de.

\section{ZBW - Leibniz-Informationszentrum Wirtschaft}

Die ZBW - Leibniz-Informationszentrum Wirtschaft (ZBW) ist die weltweit größte Informationsinfrastruktur für die Wirtschaftswissenschaften. Die Einrichtung beherbergt rund 4 Millionen Medieneinheiten und ermöglicht den Zugang zu Millionen wirtschaftswissenschaftlicher Online-Dokumente. Allein 2017 wurden rund 6 Millionen digitale Volltexte heruntergeladen. Daneben stellt die ZBW eine rasant wachsende Sammlung von Open-Access-Dokumenten zur Verfügung. EconStor, der digitale Publikationsserver, verfügt aktuell über 150.000 frei zugängliche Aufsätze und Working Papers. Mit EconBiz, dem Fachportal für wirtschaftswissenschaftliche Fachinformationen, können Studierende oder Forschende in über 10 Millionen Datensätzen recherchieren. Zudem gibt die ZBW die beiden wirtschaftspolitischen Zeitschriften Wirtschaftsdienst und Intereconomics heraus. Die ZBW ist eine forschungsbasierte wissenschaftliche Bibliothek. Drei Professuren und eine internationale Doktorandengruppe beschäftigen sich transdisziplinär mit dem Thema Digitalisierung der Wissenschaft. Die ZBW ist in ihrer Forschung international vernetzt. Hauptsächliche Kooperationspartner kommen aus EU-Großprojekten, aus DFG-Projekten sowie aus dem LeibnizForschungsverbund Science 2.0. Die ZBW ist Teil der Leibniz-Gemeinschaft und Stiftung des öffentlichen Rechts. Sie wurde mehrfach für ihre innovative Bibliotheksarbeit mit dem internationalen LIBERAward ausgezeichnet. 2014 wurde die ZBW zur „Bibliothek des Jahres“ gekürt.

Mehr Informationen unter www.zbw.eu.

\section{Kontakt:}

Ulrike Ostrzinski

Pressesprecherin ZB MED

Tel.: (0221) 478 5687, E-Mail: pressestelle@zbmed.de 


\section{Pilotprojekt zur Fernleihe von E-Books gestartet}

Das Hochschulbibliothekszentrum des Landes Nordrhein-Westfalen (hbz), der Karger Verlag und ZB MED - Informationszentrum Lebenswissenschaften starten ein Pilotprojekt zur Fernleihe von E-Books. Mit dieser Zusammenarbeit beschreiten die drei Kooperationspartner einen neuen Weg bei der überregionalen Literaturversorgung durch die Fernleihe.

ZB MED, hbz und Karger ermöglichen mit dem Pilotprojekt die Nutzung von E-Books per Fernleihe. Das hbz stellt dazu die technische Infrastruktur bereit, die auf einer vom Bibliotheksverbund Bayern entwickelten Lösung basiert.

ZB MED und Karger haben sich auf eine Lizenz verständigt, welche sowohl den lokalen Zugriff der E-Books in der Bibliothek vorsieht, als auch das Recht auf Fernleihe beinhaltet - deutschlandweit und für eine unbeschränkte Anzahl an Ausleihen. Dabei stellt ZB MED, die Zentrale Fachbibliothek für die Lebenswissenschaften für ganz Deutschland, die bei Karger lizenzierten Titel jeder anderen Bibliothek zur Verfügung, die zur überregionalen Fernleihe über die digitale Bibliothek DigiBib zugelassen ist.

Der Karger Verlag, mit Hauptsitz in Basel (Schweiz) und weiteren Niederlassungen weltweit, unterstützte das Pilotprojekt von Anfang an. „Nicht nur Bibliotheken, sondern auch Verlage müssen ihr klassisches Geschäftsmodell an den digitalen Fortschritt anpassen. Mit ZB MED haben wir einen innovativen Partner gefunden, mit dem wir uns gemeinsam diesen Herausforderungen stellen und einen direkten Austausch herstellen können, “ sagt Joachim Flickinger, Managing Director S. Karger GmbH in Freiburg, Deutschland.

Die Fernleihe dient seit jeher der Deckung des Informationsbedarfs über die lokale Grenze einer Bibliothek hinweg. Bücher oder Zeitschriftenkopien werden zwischen den Bibliotheken verschickt und stehen den Nutzenden so überregional zur Verfügung. Heute kaufen Bibliotheken jedoch vermehrt E-Books, die üblicherweise von den Verlagen lediglich für die Nutzung vor Ort lizenziert werden. Sie stellten bislang häufig eine Lücke in der deutschlandweiten Literaturversorgung dar. Mit dem neuen Angebot der drei Kooperationspartner sind E-Books jetzt in der digitalisierten Gegenwart angekommen. Das Pilotprojekt läuft bis zum 31.12.2020, danach ist eine Evaluierung durch ZB MED und Karger vorgesehen. 


\section{Hintergrund-Informationen}

\section{Das Hochschulbibliothekszentrum des Landes Nordrhein- Westfalen (hbz)}

Das Hochschulbibliothekszentrum des Landes Nordrhein-Westfalen (hbz) steht im Bereich des Bibliotheks- und Informationswesens für Wissen, Information und Innovation. Im Mittelpunkt des Aufgabenspektrums steht die bedarfsgerechte Planung und Umsetzung von Projekten in enger Absprache mit den Kunden. Auf dieser Basis wird an der konstanten Weiterentwicklung bestehender Systeme, deren Leistungsfähigkeit und Stabilität gearbeitet. Das hbz greift innovative Tendenzen und aktuelle Entwicklungsansätze auf und bietet hierfür praktikable Lösungen an. Die Verbunddatenbank des hbz weist über 20 Millionen Titel- und über 46 Millionen Bestandsnachweise nach und integriert die Nordrhein-Westfälische Bibliographie sowie alle Zeitschriften der Zeitschriftendatenbank (ZDB). Das hbz betreibt darüber hinaus DigiBib - Die Digitale Bibliothek, die von über 275 Kunden deutschlandweit genutzt wird. Weitere Produkte und Projekte des hbz: Konsortiale Erwerbung von elektronischen Inhalten, DigiLink, DigiAuskunft, die Open-Access-Plattform Digital Peer Publishing (DiPP), Bibliotheksstatistik, der Linked-Open-DataService lobid, Digitalisierung, Langzeitarchivierung, Online-Fernleihe, das OLE-/FOLIO-Evaluierungsprojekt sowie der Aufbau einer OER World Map und Dokumentlieferdienste. Zudem übernimmt das hbz die Speicherung und Verwaltung von digitalen Objekten aller Art, Hosting und Archivierung von Webseiten, die Langzeitarchivierung elektronischer Pflichtexemplare, Hosting von Hochschulschriften und Dokumentenservern.

Mehr Informationen unter https://www.hbz-nrw.de.

\section{Karger Verlag}

Der Karger Verlag ist der größte Verlag für Medizin und Naturwissenschaften in der Schweiz. Mit Sitz in Basel, Schweiz, ist Karger mit 250 Mitarbeitenden in 15 Ländern vertreten. Seit dem Gründungsjahr 1890 wird der Verlag in vierter Generation als unabhängiges Familienunternehmen geführt. Sein Ziel ist es, die Wissenschaftswelt mit qualitativ hochwertigen Publikationen in allen Fachgebieten der Medizin zu unterstützen. Das Verlagsprogramm umfasst 50 Bücher im Jahr und über 100 begutachtete Zeitschriften, darunter eine wachsende Zahl OpenAccess-Zeitschriften. Inhaltlich bilden Grundlagen- und klinische Forschung den 
Schwerpunkt. Die Publikationen erscheinen mehrheitlich auf Englisch und sind weitgehend online verfügbar.

Mehr Informationen unter www.karger.com.

\section{ZB MED - Informationszentrum Lebenswissenschaften}

ZB MED - Informationszentrum Lebenswissenschaften versteht sich als zentrale lebenswissenschaftliche Informationsinfrastruktur für Deutschland und Europa und als treibende Kraft bei der Schaffung einer vernetzten digitalen Wissensbasis. Aufbauend auf seinen einzigartigen Beständen bietet ZB MED forschungsbasierte Möglichkeiten zur Gewinnung von Informationen und Nutzung von Forschungsdaten in den Lebenswissenschaften. Als Dienstleister für die lebenswissenschaftliche Forschung ist ZB MED Partner für alle, die innovative Wege zur verbesserten Gewinnung, Verarbeitung und Nutzung von wissenschaftlichen Informationen und Forschungsdaten beschreiten wollen. Dazu bietet das Informationszentrum Literatur, Fachinformationen sowie darauf aufbauende Mehrwertdienste vor allem in digitaler Form über das semantikbasierte Suchportal LIVIVO, über das Publikationsportal PUBLISSO sowie vor Ort in Köln und Bonn an. Im Interesse einer qualitativ hochwertigen Versorgung mit wissenschaftlichen Informationen gehört die Förderung von Open Access zu den zentralen Prioritäten von ZB MED. Zudem betreibt das Informationszentrum anwendungsorientierte Forschung im Bereich „Knowledge Discovery“.

Mehr Informationen unter https://www.zbmed.de.

\section{Kontakt:}

Tiziane Schön

Marketing / Öffentlichkeitarbeit hbz

Tel.: (0221) 400 75-138, E-Mail: schoen@hbz-nrw.de

Cora Wirtz

Corporate Communications Manager, Karger

Tel.: +41 (0) 6130612 71, E-Mail: c.wirtz@karger.com

Ulrike Ostrzinski

Pressesprecherin ZB MED

Tel.: (0221) 478 5687, E-Mail: pressestelle@zbmed.de 


\section{Wertvolle Bücher brauchen Schutz}

\section{Universitätsbibliothek Frankfurt investiert 150.000 Euro in den Erhalt des schriftlichen Kulturgutes}

Die Universitätsbibliothek der Goethe-Universität Frankfurt am Main hat mehrere Projekte zum Erhalt des schriftlichen Kulturgutes angestoßen und erhält dafür große Fördersummen durch die Bundesregierung und das Land Hessen. Zusammen mit Eigenmitteln der Bibliothek wurden im laufenden Jahr 150.000 Euro in den Schutz des historischen Erbes investiert.

Die Universitätsbibliothek Johann Christian Senckenberg (UB JCS) hat im Jahr 2018 umfangreiche Mittel für den Erhalt des schriftlichen Kulturgutes eingeworben, um verschiedene Projekte zur Säuberung, Schutzverpackung und Restaurierung wertvoller einzigartiger Bestände sowie Maßnahmen gegen den Papierzerfall zu ermöglichen. Die Beauftragte der Bundesregierung für Kultur und Medien, Prof. Monika Grütters, fördert die Projekte der UB JCS mit insgesamt 48.000 Euro aus den Sondermitteln des Bundes für die Erhaltung des schriftlichen Kulturgutes (Koordinierungsstelle für die Erhaltung des schriftlichen Kulturgutes, KEK). Eine fast ebenso große Fördersumme von insgesamt 43.000 Euro kommt aus dem 2018 erstmalig durch das Hessische Ministerium für Wissenschaft und Kunst aufgelegten Landesprogramm zum Erhalt des schriftlichen Kulturgutes in Hessen. Auch zwei private Stiftungen unterstützten die Arbeiten durch eine namhafte finanzielle Zuwendung von zusammen 27.000 Euro: die Frankfurter Bürgerstiftung und die Cronstett- und Hynspergische evangelische Stiftung zu Frankfurt am Main. Einen Eigenanteil von rund 31.000 Euro bringt die Bibliothek selbst auf, somit beträgt das finanzielle Gesamtvolumen der Projekte etwa 150.000 Euro.

Die Projekte betreffen insgesamt rund 8.000 Bände Kulturgut von nationaler und internationaler Bedeutung. Dazu gehört die Sammlung der Judaica und Hebraica, darunter auch die besonders seltenen jiddischen Drucke, sowie weitere wertvolle Bände des 16. bis 19. Jahrhunderts: z. B. Drucke aus der Königlichen Gartenbibliothek Herrenhausen, Werke Maria Sibylla Merians, die Bibliotheken Arthur Schopenhauers und der Frankfurter Patrizierfamilie von Holzhausen. Außerdem lässt die Universitätsbibliothek die historischen Ausgaben der bis 1943 erschienenen „Frankfurter Zeitung“ gegen den drohenden säurebedingten Papierzerfall behandeln. Diese Zeitung gilt als Vorgängerin der heutigen „Frankfurter Allgemeinen Zeitung “ und hatte in ihrer Zeit eine vergleichbare Bedeutung. Insgesamt liegt ein Schwerpunkt der Maßnahmen auf der Reinigung wertvoller Bücher und ihrer Ausstattung mit Schutzverpackungen, welche durch eine Spezialfirma maßangefertigt werden. Damit sind die Bücher zukünftig optimal vor 
mechanischen Schäden, eventuellen klimatischen Schwankungen und Schädlingsbefall geschützt.

Die UB JCS verfolgt in den letzten Jahren verstärkt Maßnahmen, um die Lagerungsbedingungen für ihre historischen Bestände nachhaltig und entsprechend neusten wissenschaftlichen Standards zu verbessern. Ein wichtiger Schritt in diesem Prozess war im Vorjahr die Einrichtung eines neuen Rara-Magazins für besonders wertvolle Drucke. Zuletzt wurden außerdem die Zuständigkeiten in diesem Bereich in einer neu strukturierten Abteilung „Bestandserhaltung und Digitalisierung“ zusammengeführt.

\section{Kontakt:}

Dr. Mathias Jehn

Leiter der Abteilung Bestandserhaltung und Digitalisierung sowie des Archivzentrums und der Sammlung Frankfurt und Seltene Drucke

Universitätsbibliothek J. C. Senckenberg

Bockenheimer Landstraße 134-138, 60325 Frankfurt am Main

Tel.: (069) 798 39007, E-Mail: m.jehn@ub.uni-frankfurt.de

\section{Kulturerbe digital: Die Badische Landesbibliothek stellt in ihren Digitalen Sammlungen das dreimillionste Bild online}

Seit 2010 digitalisiert die Badische Landesbibliothek herausragende Bestände ihrer umfangreichen historischen Sammlungen, um sie der Öffentlichkeit kostenlos in den Digitalen Sammlungen zur Verfügung zu stellen. Kurz vor Jahresende hat die Digitalisierungswerkstatt der Badischen Landesbibliothek nun einen weiteren Meilenstein erreicht und die dreimillionste Seite digitalisiert. „Wir freuen uns sehr, dass wir das vielfältige digitale Angebot der Badischen Landesbibliothek konsequent erweitern und nun bereits das dreimillionste Bild online stellen können, das aus einem im 16. Jahrhundert entstandenen Straßburger Wappenbuch stammt. Dies ist ein wunderbarer Erfolg unserer Bemühungen, auf den wir sehr stolz sind“, kommentiert die Leitende Bibliotheksdirektorin Dr. Julia von Hiller.

Die Handschrift, aus der das Bild stammt, gehört zu den wenigen Büchern aus der Bibliothek der Ortenauer Reichsritterschaft in Offenburg, welche die Mediati- 
sierung zwischen 1803 und 1806 überstanden haben und seit dem 19. Jahrhundert in Karlsruhe bewahrt werden. Angelegt um 1594/95, wurde das Straßburger Wappenbuch noch bis ins 18. Jahrhundert hinein mit handschriftlichen Eintragungen ergänzt. Es zeigt die Wappen sämtlicher namentlich genannter „Stettmeister“ und „Ammeister“ der Stadt Straßburg in chronologischer Reihenfolge. In der besonderen politischen Ordnung der Stadt, die seit dem 15. Jahrhundert eine Art Gewaltenteilung mit mehreren Räten vorsah, stellten diese beiden Ämter die führende exekutive Instanz dar.

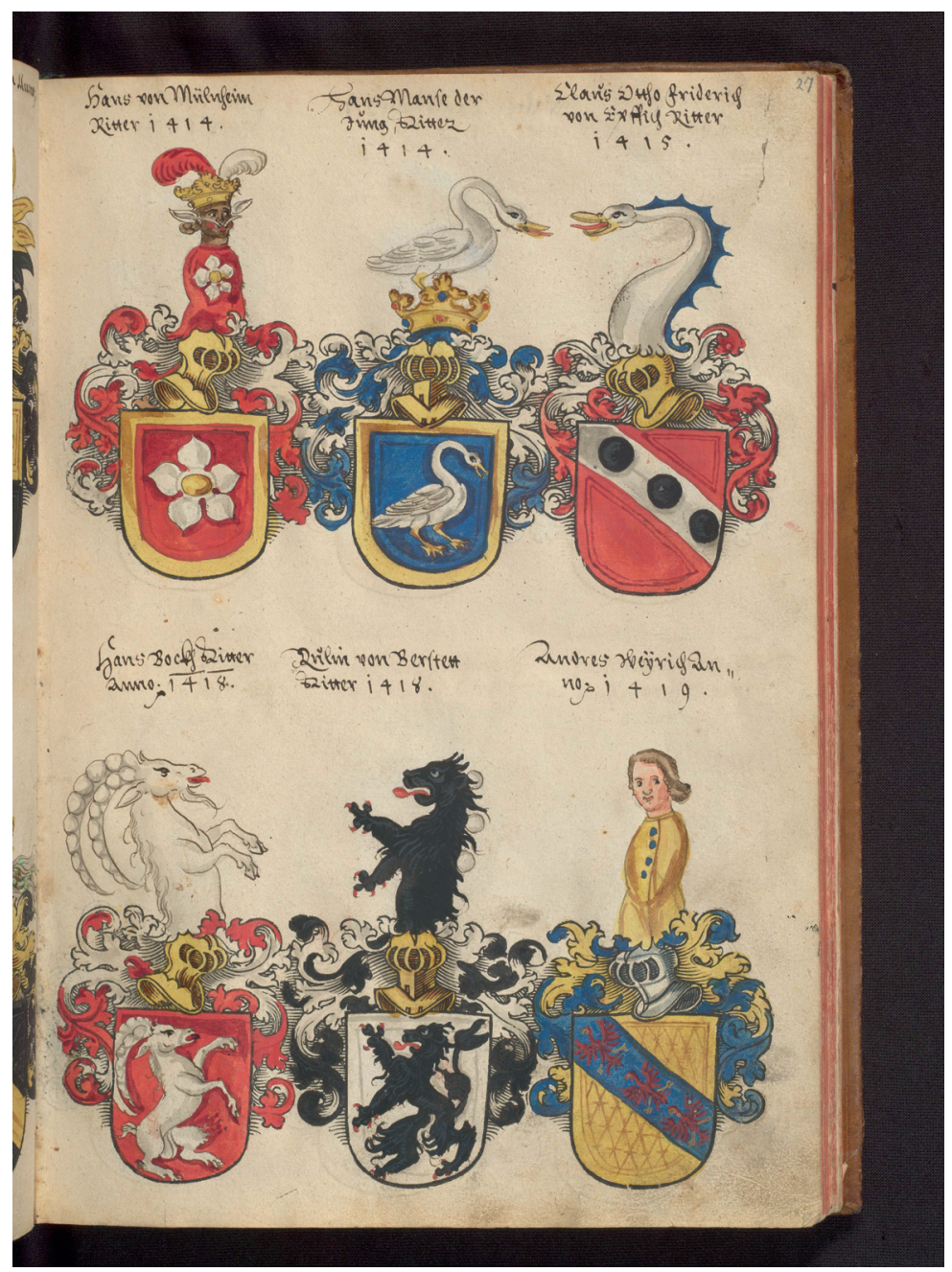

Abb. 1: Foto: Badische Landesbibliothek 
Die Badische Landesbibliothek wählt jedes Jahr bestimmte Segmente ihres einzigartigen Bestandes für umfangreiche Digitalisierungsprojekte aus, um das nach Handschriften, Musikalien und Regionalia gegliederte Angebot ihrer Digitalen Sammlungen kontinuierlich auszubauen. Die Präsentation der digitalisierten Bestände wird dabei stetig weiterentwickelt, um den Besuchern vielfältige und bequeme Nutzungsmöglichkeiten anbieten zu können.

\section{Ein neues Angebot in den Digitalen Sammlungen der Badischen Landesbibliothek: Kompositionen der Karlsruher Musikerin Clara Faisst}

Allen Musikinteressierten bieten die Digitalen Sammlungen der Badischen Landesbibliothek ab sofort ein neues Angebot zu der bekannten Karlsruher Komponistin, Musikpädagogin, Pianistin und Dichterin Clara Faisst (1872-1948), von deren Nachlass ein Teil in der Badischen Landesbibliothek bewahrt wird. Clara Faisst hat vorwiegend Lieder komponiert, die auch in großer Zahl veröffentlicht worden sind. Bei ihren späteren Werken handelt es sich hauptsächlich um Instrumentalmusik. 70 Jahre nach dem Tod der Komponistin wurden diese Werke nun digitalisiert und stehen ab sofort unter https://digital.blb-karlsruhe.de/topic/ view/4976308 zur Verfügung.

Ihre musikalische Ausbildung erhielt Clara Faisst zunächst am Großherzoglichen Konservatorium in Karlsruhe. Im Jahr 1894 ging sie zum Studium nach Berlin an die Königliche Hochschule für Musik. Dort war sie Schülerin von Ernst Rudorff (1840-1916), Robert Kahn (1865-1951), Woldemar Bargiel (1828-1897) und Max Bruch (1838-1920), an dessen Meisterklasse für Komposition sie teilnahm. Im Anschluss an ihr Studium kehrte Clara Faisst nach Karlsruhe zurück, wo sie als Künstlerin und Lehrerin wirkte.

Die Musikaliensammlung der Badischen Landesbibliothek gehört zu den größten und bedeutendsten des Landes Baden-Württemberg. Sie ist über Jahrhunderte hinweg gewachsen und reicht von mittelalterlichen Musikhandschriften bis zur Hofmusik badischer Residenzen. Ergänzt wird der Bestand durch zahlreiche Nachlässe von Komponisten und Musikern, die über die regionale Musikgeschichte hinaus von großem Stellenwert sind. Schrittweise werden 
besonders interessante Teile dieses einzigartigen historischen Bestandes digitalisiert, um sie der Öffentlichkeit kostenlos zur Verfügung zu stellen.

\section{Weitere Informationen im Internet:}

Digitale Sammlungen der Badischen Landesbibliothek: https://digital.blb-karlsruhe.de

\section{Kontakt:}

Badische Landesbibliothek und Badische Bibliotheksgesellschaft Pressereferat / Karen Evers M.A.

\section{Der Donaueschinger Wigalois - ein Artusritter kehrt zurück}

Mit großzügiger Unterstützung der Ernst von Siemens Kunststiftung, der Kulturstiftung der Länder, der Beauftragten der Bundesregierung für Kultur und Medien und der Wüstenrot Stiftung konnte die Badische Landesbibliothek Ende 2018 die ehemals Donaueschinger Wigalois-Handschrift (Cod. Don. 71) erwerben, ein nationales Kulturdenkmal von exzeptionellem Wert. Damit kehrt ein um 1420 in der berühmten Lauber-Werkstatt im oberrheinischen Hagenau produzierter und höchst erzählfreudig illustrierter Artusroman in seinen ursprünglichen Sammlungs- und Überlieferungskontext zurück.

Kulturstaatsministerin Prof. Monika Grütters hat sich gern für den Kauf engagiert: „Die Donaueschinger Wigalois-Handschrift nimmt nicht nur in der deutschen Kunst- und Kulturgeschichte eine herausragende Stellung ein. Sie ist auch für das europäische Kulturerbe von außergewöhnlicher Bedeutung. Es ist ein schöner Erfolg, dass es uns - auch mit der Bereitstellung erheblicher Bundesmittel - gelungen ist, diese einzigartige deutschsprachige Bilderhandschrift des Mittelalters für die Badische Landesbibliothek Karlsruhe zu erwerben. Damit findet dieses wertvolle Kulturerbe in seinen ursprünglichen Sammlungs- und Überlieferungskontext zurück.“

Dr. Martin Hoernes, Generalsekretär der Ernst von Siemens Kunststiftung, freut sich: „Der Erwerb des bedeutenden und illustrierten Artusromans steht 


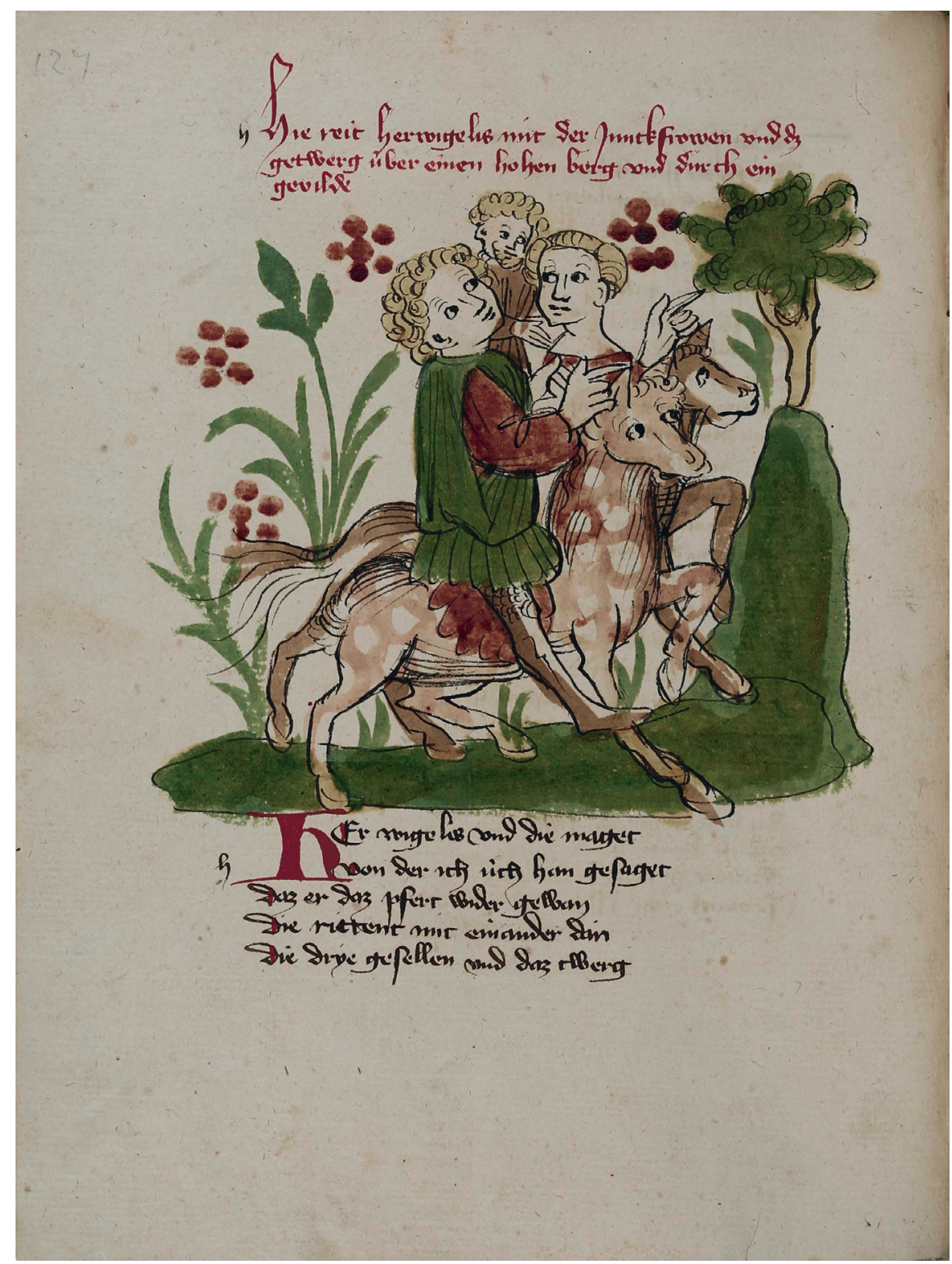

Abb. 2: Bl. 64v: Wigalois und Elamie, die Königin von Tyrus, der er im Kampf gegen den Roten Ritter zu ihrem Recht verholfen hat, reiten mit dem Zwerg weiter nach Roimunt. 
exemplarisch für die Fördertradition der Ernst von Siemens Kunststiftung: Dank des Vermächtnisses unseres Gründers und Mitteln der Siemens AG konnte ein einzelne Institutionen überfordernder Jahrhundertankauf abgeschlossen werden. Die wertvolle Handschrift von nationalem Rang kann nun in die Nähe ihres Entstehungsortes und an die Öffentlichkeit zurückkehren.“

Prof. Dr. Frank Druffner, Stv. Generalsekretär der Kulturstiftung der Länder, ergänzt: „Bei dieser Handschrift handelt es sich um eine Rarität, die eine zentrale Station der deutschen, mithin der europäischen Kulturgeschichte dokumentiert. Denn sie stammt aus einer Werkstatt, die Klassiker-Ausgaben auf Vorrat produzierte und somit das Verlagswesen der Buchdruck-Epoche vorwegnahm. Mit der Erwerbung kehrt sie zurück in die Region, aus der sie stammt, und in eine Sammlung, deren Teil sie war. Damit steht sie nach mehr als 25 Jahren wieder der kunsthistorischen, kulturhistorischen, literaturwissenschaftlichen und bildungsgeschichtlichen Forschung zur Verfügung, für die sie von außerordentlichem Wert ist.“

Prof. Philip Kurz, Geschäftsführer der Wüstenrot Stiftung, hat sich von der großen Seltenheit des Angebots deutschsprachiger Handschriften des Mittelalters im Generellen und der besonderen Rarität überlieferter weltlich-literarischer Texte mit Illustrationen im Speziellen für ein Engagement seiner Stiftung begeistern lassen: „Mit der gemeinsamen Erwerbung der prächtig bebilderten WigaloisHandschrift ist herausragendes kulturelles Erbe wieder nach Baden-Württemberg zurückgeholt worden. Unter besten Bedingungen steht der Wigalois in der Badischen Landesbibliothek für Forschung und Öffentlichkeit endlich wieder zur Verfügung.“

\section{Hintergrundinformationen zum Donaueschinger Wigalois}

Um das Jahr 1215 erzählte der fränkische Dichter Wirnt von Grafenberg in diesem mittelhochdeutschen Versroman die Geschichte des Titelhelden Wigalois, der am Hof des Königs Artus zum Ritter ausgebildet wird. Von dort bricht er auf, um das Reich Korntin von seinem Usurpator Roaz zu befreien und seiner rechtmäßigen Königin Larie zurückzugeben.

Die Handschrift entstand um 1420 im elsässischen Hagenau. Hier produzierten Schreiber und Illustratoren repräsentative „Klassiker-Ausgaben“ der deutschen Literatur auf Vorrat für einen Käufermarkt. Daraus entwickelte sich die Werkstatt des Diebold Lauber als großes kommerzielles Unternehmen, das zwischen 1427 und 1471 nachweisbar ist und erst zu Beginn der Buchdruck-Ära 
einging. In zwei seiner Bücheranzeigen wird unter den lieferbaren Handschriften ein bebilderter Wigalois aufgeführt.

Die herausragende kunst- und kulturhistorische Bedeutung der Handschrift gründet auf ihrer lebhaften Illustration, die den Text höchst erzählfreudig visualisiert und aufschlussreich interpretiert. Enthalten sind 30 (von ehemals 31) halbbis ganzseitige farbige Federzeichnungen von der Hand eines einzigen Zeichners in Grün-, Rot-, Gelb- und Brauntönen, die die Geschichte schwungvoll und vergnügt in Szene setzen.

Die Hagenauer Schreibwerkstatt, berühmt für die hohe Qualität ihrer Handschriften in Bildausstattung und Textbeschaffenheit, hat wesentlichen Anteil an der Überlieferung jener literarischen Texte des Hochmittelalters, die im 15. Jahrhundert in verändertem gesellschaftlichem Kontext noch einmal neu und anders rezipiert wurden. Käufer eines Artusromans von kanonischem Rang vergewisserten sich durch den Besitz einer solchen Handschrift der ideellen Teilhabe am überlieferten ritterlichen Ethos und daraus folgend des Anspruchs auf eine führende gesellschaftliche Stellung.

Die Handschrift fand wahrscheinlich schon im 15. Jahrhundert einen adligen Käufer am Oberrhein. Sie gehörte im 18. Jahrhundert zum Grundstock der berühmten Fürstlich Fürstenbergischen Hofbibliothek in Donaueschingen. Seit der Erwerbung der Donaueschinger Handschriftensammlung durch das Land Baden-Württemberg 1993 war es Ziel, den vorab in Privatbesitz verkauften Codex für die Sammlung zurückzugewinnen. Er hat nun seinen angestammten Platz neben dem „Parzival“ Wolframs von Eschenbach wieder eingenommen. Die Badische Landesbibliothek ist ihren Förderern unendlich dankbar, dass sie die Rückkehr der Handschrift in ihren ursprünglichen Sammlungs- und Überlieferungszusammenhang ermöglicht haben.

Mehr Informationen unter https://digital.blb-karlsruhe.de/wigalois.

\section{Kontakt:}

Badische Landesbibliothek

Dr. Julia Freifrau Hiller von Gaertringen

Ltd. Bibliotheksdirektorin

Erbprinzenstraße 15, 76133 Karlsruhe

Telefon: +49 721 175-2201, Fax: +49 721 175-2333,

E-Mail: direktion@blb-karlsruhe.de 


\title{
Mercator, Kopernikus und Cellarius
}

\author{
Staatsbibliothek setzt Ausstellungstrilogie „Gott, die Welt \\ und Bayern" mit kartografischen und wissenschaftlichen \\ Glanzstücken fort
}

Ab 21. Januar 2019 zeigt die Bayerische Staatsbibliothek im zweiten Teil ihrer Ausstellung „Gott, die Welt und Bayern“ Bücher, Karten und Globen des 16. und 17. Jahrhunderts. Unter dem Titel „Aus Orient und Okzident“ sind unter anderem Glanzstücke wie zwei Globen von Gerhard Mercator, der Himmelsatlas von Andreas Cellarius, „De Revolutionibus“ des Nikolaus Kopernikus sowie ein Wappen von Lucas Cranach d. Ä. zu sehen.

$\mathrm{Zu}$ Beginn der Frühen Neuzeit weitete sich das Weltbild, neue Seewege und unbekannte Kontinente wurden entdeckt und in Karten und Globen vermerkt. Das Fragment einer Weltkarte für das Handelshaus der Welser in Augsburg von 1530 zeigt, dass Kaufleute die Tragweite dieser neuen Erkenntnisse schnell erkannten. $\mathrm{Zu}$ sehen sind auch prächtig gestaltete Einbände von Werken über fremde Welten und Kulturen aus den Privatbibliotheken humanistisch gebildeter Gelehrter und Fürsten. Das 16. Jahrhundert war auch ein Jahrhundert des wissenschaftlichen Aufbruchs: Nikolaus Kopernikus’ Abhandlung „De Revolutionibus Orbium Coelestium“ über seine umwälzende Erkenntnis, dass die Sonne und nicht die Erde im Zentrum des damals bekannten Universums steht, wird ebenso gezeigt wie der prächtig illustrierte Himmelsatlas von Andreas Cellarius oder Gerhard Mercators detailreiche Erd- und Himmelsgloben (1541).

Die Ausstellung „Gott, die Welt und Bayern - 100 Kostbarkeiten aus den regionalen Staatlichen Bibliotheken Bayerns“ präsentiert von 17. Oktober 2018 bis 7. Juli 2019 zum ersten Mal vereint in der Bayerischen Staatsbibliothek in München hundert Bestandshighlights der zehn regionalen Staatlichen Bibliotheken in Bayern.

Die zehn regionalen Staatlichen Bibliotheken Bayerns sind der Bayerischen Staatsbibliothek in München unmittelbar nachgeordnet. $\mathrm{Zu}$ ihnen zählen die Staatlichen Bibliotheken in Amberg, Ansbach, Neuburg an der Donau, Passau und Regensburg sowie die Hofbibliothek Aschaffenburg, die Staats- und Stadtbibliothek Augsburg, die Staatsbibliothek Bamberg, die Landesbibliothek Coburg und die Studienbibliothek Dillingen.

Als wissenschaftliche Bibliotheken dienen sie der Informations- und Literaturversorgung ihrer Region sowie der Sicherung, Erhaltung und Pflege wertvoller historischer Bestände. Die Bibliotheken bewahren wertvolle Handschriften und Frühdrucke, herausragende Sondersammlungen und bedeutende Nachlässe. Sie 
erfüllen eine wesentliche kulturpolitische Funktion, indem sie für jeweils eine bestimmte Region zuständig sind und Publikationen, die dort entstanden sind oder einen inhaltlichen Bezug zur Region haben, möglichst vollständig sammeln und für die Öffentlichkeit zur Nutzung bereitstellen.

\section{Teile zwei und drei des dreiteiligen Ausstellungszyklus:}

- 21. Januar 2019 - 7. April 2019: Aus Orient und Okzident. Bücher, Karten, Globen des 16. und 17. Jahrhunderts.

- $\quad$ 15. April 2019 - 7. Juli 2019: Krieg und Frieden, Freud und Leid. Sammelobjekte des 17. bis 20. Jahrhunderts.

\section{Weitere Informationen:}

- Website mit Informationen zur Ausstellung, zu Führungen und zum Begleitprogramm: www.gott-welt-bayern.de

- Öffnungszeiten: Montag bis Freitag 11 - 18 Uhr, Sonntag 13 - 17 Uhr. An Feiertagen geschlossen. Eintritt frei.

- Katalog: Zur Ausstellung erscheint ein Katalog zum Preis von $19 €$ (Verkaufspreis vor Ort in der Ausstellung). Mit freundlicher Unterstützung der Förderer und Freunde der Bayerischen Staatsbibliothek e.V.

- Ort: Bayerische Staatsbibliothek, Ludwigstr. 16, 80539 München, U3/6, Bus 58/68/153/154 Haltestelle Universität, Bus 100/153 Haltestelle Von-der-TannStraße.

\section{Kontakt:}

Dr. Bettina Wagner

Staatsbibliothek Bamberg

Tel.: +49 951 95503-112, E-Mail: bettina.wagner@staatsbibliothek-bamberg.de

Dr. Bernhard Lübbers

Staatliche Bibliothek Regensburg

Tel.: +49941 630806-0, E-Mail: b.luebbers@sb-regensburg.de

Peter Schnitzlein

Presse- und Öffentlichkeitsarbeit, Bayerische Staatsbibliothek

Tel.: +49 89 28638-2429, E-Mail: presse@bsb-muenchen.de 